\title{
Reconstruction d'un modèle CAO à partir d'un maillage déformé
}

\section{Application dans le cas de grands déplacements}

\author{
Borhen Louhichi ${ }^{1, a}$, Mehdi Tlija ${ }^{1}$, Abdelmajid BenAmara ${ }^{1}$ et Vincent François ${ }^{2}$ \\ 1 Laboratoire de Génie Mécanique LGM, École Nationale d'Ingénieurs de Monastir, 5 Av. Ibn Eljazzar, 5019 Monastir, Tunisie \\ ${ }^{2}$ Département de Génie Mécanique, Université du Québec à Trois-Rivières CP 500, Trois-Rivières, Québec, G9A 5H7, Canada
}

Reçu le 8 septembre 2009, accepté le 10 septembre 2009

\begin{abstract}
Résumé - De nos jours, nous assistons à un développement du travail en mode projet qui se caractérise par la mobilisation de compétences multiples. La globalisation des marchés ainsi que la réduction des coûts et des délais de développement de nouveaux produits ont conduit à la mise au point d'outils de travail collaboratif assurant la structuration, le suivi, et la traçabilité des échanges. Cela a induit un accroissement considérable des besoins de communication inter-applications et de cohérence globale des systèmes supports des différents modèles du produit (CAO, calcul, FAO). De part leur forte interdépendance, les deux activités $\mathrm{CAO}$ et calcul seront donc amenées à s'édifier sur de nouvelles technologies émergentes dans les domaines de la modélisation des données (modèle de produit) et des processus (interopérabilité) afin de pouvoir prendre en compte la manipulation d'objets hétérogènes (géométrie, sollicitations, maillage, déformation, etc.). En effet, dans un contexte de travail collaboratif, l'intégration numérique de ces deux activités CAO et calcul, est devenue une des principales préoccupations en CFAO. L'objectif recherché est de favoriser le partage des données sans recopies ou transformations manuelles afin de fluidifier les flux d'informations entre CAO et calcul tout en garantissant la fiabilité et la traçabilité des données. L'objet de cet article est de présenter une approche d'intégration numérique et d'interopérabilité des processus de CAO et de calcul mécanique qui consiste à retrouver le modèle CAO déformé à partir d'un maillage déformé dans la démarche de retour des résultats de calcul éléments-finis vers la CAO.
\end{abstract}

Mots clés : Intégration / simulation / calcul / reconstruction / CAO / BREP

\begin{abstract}
Rebuilding of CAD model from deformed mesh important displacement case. Nowadays, we attend a development of work in project mode which is characterized by the mobilization of multiple competences. The globalisation of the markets as well as the reduction in costs and in delays for developing new products led to the development of collaborative working tools in a distant way ensuring the structuring, the follow-up, and the traceability of the exchanges. That induced a considerable increase in the needs of inter-applications communication and total coherence of the support systems of the various product models (CAD, analysis, CAM). Besides their strong interdependences, both CAD and calculation activities will be built on new emergent technologies in the fields of the data modeling (model of product) and of the processes (interoperability) in order to be able to take into account the handling of heterogeneous objects (geometry, meshing, deformation, etc.). Indeed, in a context of collaborative work, the numerical integration of these two activities: CAD and calculation became one of the main concerns in $\mathrm{CAD} / \mathrm{CAM}$. The required objective here is to promote sharing the data without manual transformations in order to improve the flow of information between CAD and calculation while guaranteeing the reliability and the traceability of the data. Our research is oriented to CAD/CAM/analysis integration by rebuilding the CAD model starting from the FE results (deformed mesh). This paper presents techniques for rebuilding the geometrical and topological entities of the CAD model starting from a deformed mesh.
\end{abstract}

Key words: Integration / simulation / analysis / reconstruction / CAD / BREP

a Auteur pour correspondance : lborhen@gmail.com 


\section{Introduction}

La conception d'un produit est un exercice dans lequel il s'agit de proposer des solutions ou reconcevoir des solutions existantes pour remplir des fonctions bien définies à l'intérieur d'un ensemble de contraintes. Généralement, l'obtention d'une solution n'est pas directe sauf pour des problèmes extrêmement simples. Le processus est plutôt itératif au cours duquel un objet est conçu et modifié afin qu'il puisse remplir des fonctions bien définies et se conformer à un ensemble de contraintes [1].

Dans un processus de conception, deux principales phases sont vitales et interdépendantes, à savoir la modélisation géométrique du produit $(\mathrm{CAO})$ et le calcul de dimensionnement et de validation du modèle proposé. Le calcul de dimensionnement consiste à déterminer les dimensions en fonction des sollicitations, tandis que le calcul de validation (basé sur des critères) consiste à vérifier si les dimensions choisies ou calculées permettent de résister aux sollicitations. Le calcul mécanique est réparti tout au long du cycle d'élaboration de produit et permet de déterminer au plus tôt les performances d'un produit. Pour permettre aux concepteurs de choisir des solutions technologiques et techniques alternatives par comparaison de leurs performances, il est nécessaire d'utiliser des méthodes de calcul nécessitant un délai court tout en assurant une précision des résultats suffisante pour pouvoir choisir la solution la plus performante.

En conception mécanique, la méthode de calcul la plus utilisée est la méthode des éléments-finis. Elle a l'avantage de pouvoir s'appliquer à divers types de problèmes physiques ou mécaniques. Sa formulation est basée sur la mécanique des milieux continus et est exprimée au moyen d'équations aux dérivées partielles. L'intégration de cette méthode dans les outils de CFAO d'aujourd'hui est devenue une préoccupation de la plupart des laboratoires de recherche liés au domaine de la conception et la production intégrées.

Dans un contexte d'ingénierie simultanée, les systèmes supports de l'activité de conception doivent permettre une coopération dans l'accomplissement des tâches de conception et de calcul. Cela permettra de minimiser les classiques allers-retours entre conception et calcul et réduit de manière significative le coût de la conception [2]. Dans la littérature plusieurs travaux de recherche s'intéressent à la même problématique d'intégration, à savoir : les travaux publiés par la commission de simulation numérique de MICADO, les projets SG3C, OSCAR et VIVACE [1,3-5]. Notons que la récupération des résultats de calcul (iso-valeurs, tableaux de valeurs etc.) dans l'environnement de CAO est actuellement à la charge du concepteur. Afin de remédier à ces problèmes, plusieurs travaux de recherche ont été menés dans l'objectif d'améliorer les échanges bidirectionnels entre les outils de CAO et de calcul $[6,7]$.

Ainsi, nos travaux de recherche rentrent dans l'esprit d'intégration de la méthode des éléments-finis dans le processus de CAO-FAO. L'objectif de nos recherches est de développer un outil de reconstruction du modèle CAO à partir des résultats éléments-finis (maillage déformé).
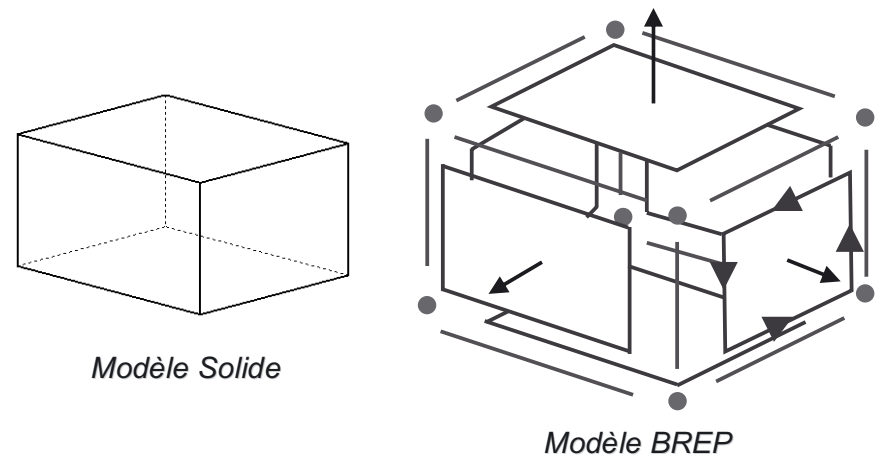

Fig. 1. Modèle BREP.

Cela donne au concepteur un moyen quantitatif (modèle CAO reconstruit en mode déformé) prêt à être injecté dans le mécanisme en vue d'une évaluation.

La reconstruction du modèle $\mathrm{CAO}$ à partir des résultats éléments-finis (maillage déformé) facilite le libre échange entre conception et calcul et rend possible la visualisation et la simulation du comportement d'un assemblage à l'état déformé (état normal de fonctionnement) afin de détecter des éventuelles interférences [8]. Actuellement les systèmes CAO traitent les assemblages dans leurs configurations rigides. De plus, la reconstruction permet la simulation en configuration réelle des processus de conception et/ou fabrication de produit mécanique (simulation du processus d'emboutissage par exemple). Autour des déformations permanentes de la pièce, le modèle CAO est reconstruit, le maillage est recalculé et les conditions aux limites sont repositionnées sur la nouvelle forme du modèle.

Les besoins industriels dans ce domaine sont très clairs : disposer d'outils rendant les simulations numériques aussi automatiques et aussi bien intégrées aux contraintes des cycles de conception que possible [9] afin de valider rapidement de nouveaux concepts et ainsi de gagner en coût, en délai et en qualité [10].

\section{Choix de modèle de reconstruction}

Les modèles de CAO se basent dans la plupart du temps sur le modèle BREP (Boundary Representation) [11]. Ce modèle est utilisé pour la reconstruction de modèle $\mathrm{CAO}$ en vue de garantir la comptabilité avec la plupart des logiciels de CAO. La modélisation BREP est basée sur les frontières (faces, contours, arêtes et sommets) (Fig. 1). Le modèle BREP est constitué d'informations topologiques et géométriques. Les informations topologiques existantes au sein d'un modèle BREP sont les connectivités, les liens et les orientations des différentes entités (faces, contours, arêtes et sommets). Les informations géométriques au sein du modèle BREP se résument dans les natures géométriques des surfaces et courbes porteuses des différentes faces et arêtes (face plane, courbe circulaire...). 


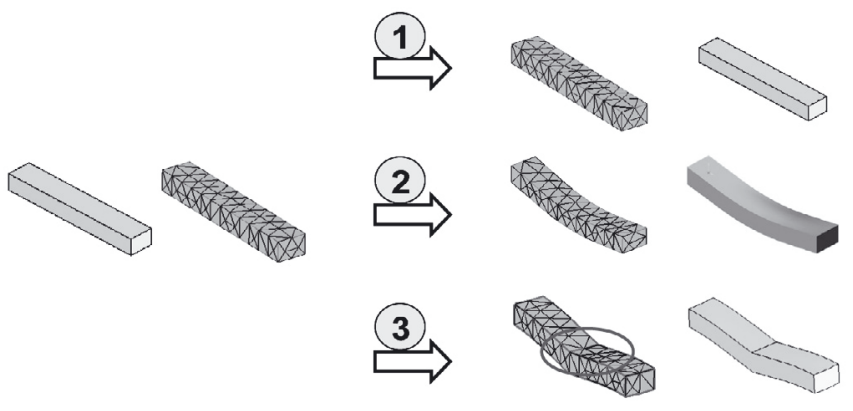

Fig. 2. Cas des déformations possibles.

Les avantages de ce modèle sont :

- ce modèle est facile en description, visualisation et transformation géométrique;

- il est unique (pour une pièce mécanique, il existe un seul modèle BREP);

- il est disponible sur la plupart des systèmes CAO.

Dans cet article, des techniques de reconstruction des entités géométriques et topologiques d'un modèle BREP à partir d'un maillage déformé issu d'un résultat de calcul sont présentées.

\section{Reconstruction d'un modèle CAO à partir des résultats d'une analyse éléments-finis : algorithme développé}

Le travail concerne l'étude de comportement des objets dans le domaine de la mécanique du solide. L'étude s'intéresse au comportement en petites et en grandes perturbations (élasticité et élasto-plasticité). Lors de la déformation d'un objet mécanique trois cas de changements topologiques et géométriques peuvent se présenter (Fig. 2) :

- déformation sans changements topologiques, ni géométriques du modèle. C'est-à-dire le nombre de faces, de contours, d'arêtes et de sommets reste le même ainsi que leurs types géométriques et leurs connectivités. Ce cas de figure se présente lors d'une étude élastique dans le cas de traction ou de compression d'une pièce mécanique ;

- déformation sans changements topologiques, mais avec des changements géométriques du modèle. C'està-dire la nature des faces, des arêtes et des sommets change tout en gardant leurs nombres et leurs connectivités. Ce cas de figure est très fréquent en mécanique, il se présente dans le cas des essais de flexion par exemple;

- déformation avec changements topologiques et géométriques du modèle. C'est-à-dire la nature, le nombre et les connectivités des entités changent. Ce cas de figure se présente lors de la propagation d'une fissure dans une pièce. Ce cas peut également se présenter dans les procédés d'emboutissage, de pliage. Dans plusieurs cas, le problème peut se retrouver

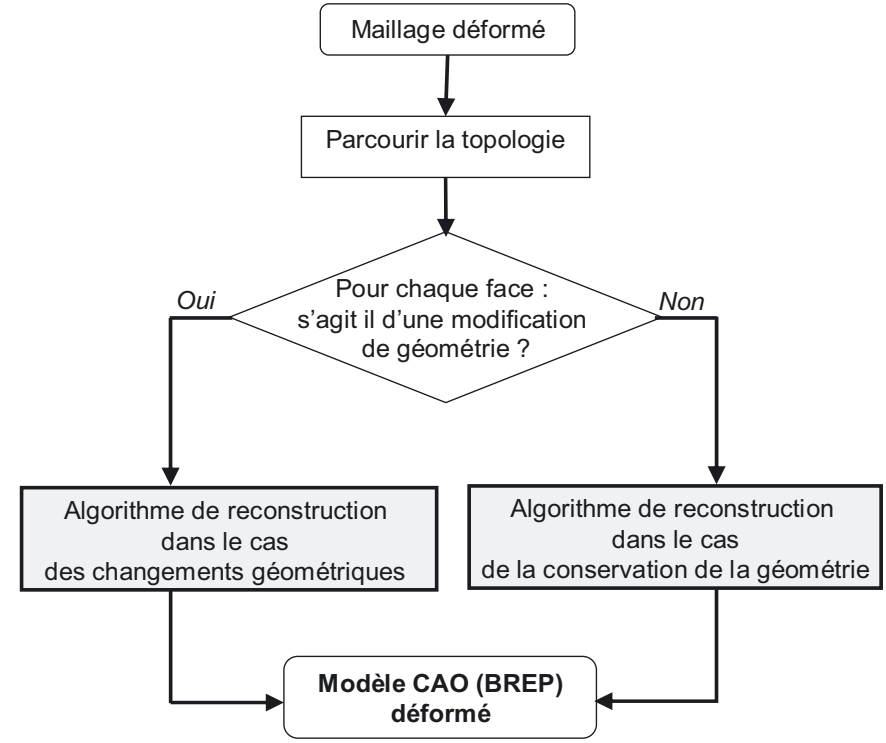

Fig. 3. Algorithme général de reconstruction.

dans le deuxième cas de déformations. En effet, pour le cas du pliage par exemple, la ligne de pliage est ajoutée dès le début (dans la CAO avant le calcul) afin de modéliser correctement les conditions aux limites. Donc la topologie est modifiée avant le calcul. Cela revient au $2^{\mathrm{e}}$ type de déformation.

Connaissant la complexité du problème de reconstruction et la diversité des cas traités en calcul par éléments-finis, l'algorithme général (Fig. 3) doit être flexible et dynamique afin d'englober les différents cas traités. Dans cet algorithme, la première phase consiste à chercher la topologie et à identifier les différentes entités géométriques du modèle BREP. La deuxième phase, consiste à reconstruire ces entités identifiées précédemment.

En termes de reconstruction, deux cas peuvent être envisagés :

- cas des petites déformations (petites perturbations) : dans ce cas, le nombre d'entités du modèle BREP ainsi que leurs natures géométriques sont connues (identiques au modèle initial). Cependant au niveau de la reconstruction, les paramétrisations (point d'insertion, rayon, vecteur directeur...) des entités géométriques sont mises à jour par rapport à celles avant déformation [8];

- cas des grandes déformations : c'est le cas le plus complexe, dans lequel les faces changent de géométrie. C'est dans cette phase que l'algorithme doit être dynamique afin d'englober les divers cas rencontrés.

D'une façon générale, l'algorithme de reconstruction suit la hiérarchie du modèle BREP (Fig. 4). Dans une première étape, on commence par l'identification de la topologie (l'identification des triangulations correspondantes aux faces). Par la suite, les surfaces porteuses des faces sont déterminées à partir des informations précédentes. Dans une troisième étape, les contours sont ajoutés aux surfaces pour obtenir les faces afin d'aboutir au modèle BREP déformé. 


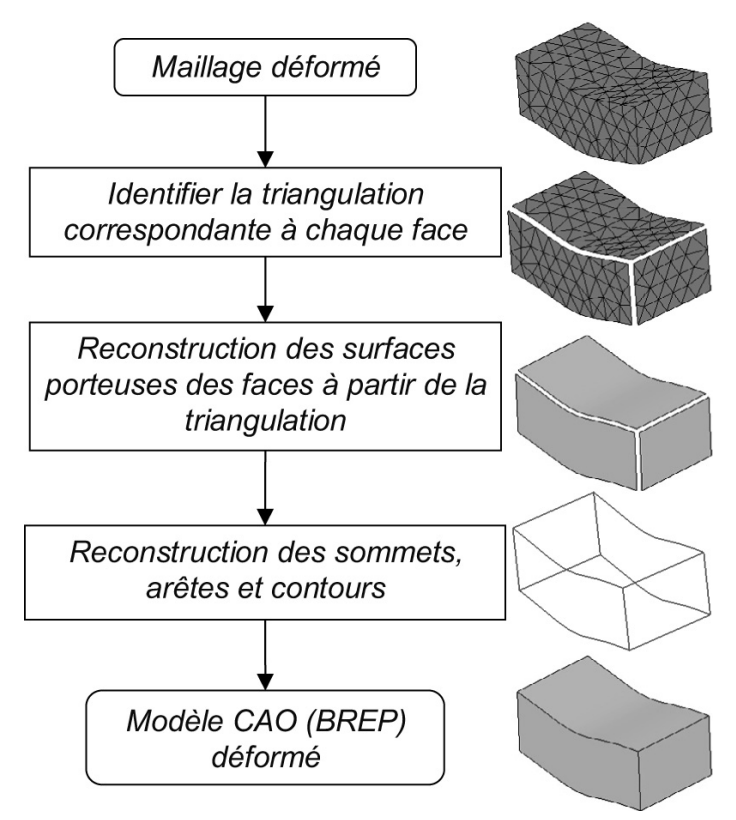

Fig. 4. Algorithme général de reconstruction.

\section{Reconstruction des entités d'un modèle BREP : état de l'art}

Le modèle BREP est une description par les frontières d'un solide. Sa reconstruction revient à reconstruire ses frontières (faces, contours, arêtes et sommets). Pour reconstruire les faces et les arêtes, il faut commencer avant tout par construire leurs surfaces et courbes supports. Ces dernières sont évaluées, paramétrées et reconstruites à partir du maillage déformé (nœuds, segments et triangles).

Depuis plusieurs années, plusieurs recherches sont faites sur la reconstruction des courbes et surfaces approximatives à partir d'un nuage de points. Kruth [12] et Yin [13] ont développé une méthode de reconstruction d'une surface à partir d'un nuage de points (dans la plupart des cas réguliers) issu d'une machine à mesure tridimensionnelle. Dans le même contexte, d'autres travaux ont développé de nouvelles techniques de paramétrisation des courbes et surfaces NURBS [14,15] à partir d'un nuage de points.

D'autres travaux sont basés sur les triangles de Bézier pour l'évaluation d'une surface à partir d'un maillage. Dans ces travaux une surface triangulaire est évaluée (Fig. 5) et calculée (triangle de Bézier) à partir de chaque triangle du maillage, de telle façon qu'elle soit continue avec les surfaces voisines (triangles voisins du maillage). La continuité est de type $C^{1}$ ou $C^{2}$ [16-20].

En 1998 Volpin [21] a développé un algorithme de reconstruction d'une surface NURBS à partir d'un maillage. La méthode est basée sur trois phases : la simplification du modèle maillé initial, la création d'un maillage quadrilatéral du modèle, la construction des surfaces lisses à partir du maillage quadrilatéral. En 2004, Sarraga [22] a développé une technique, de modification d'une surface
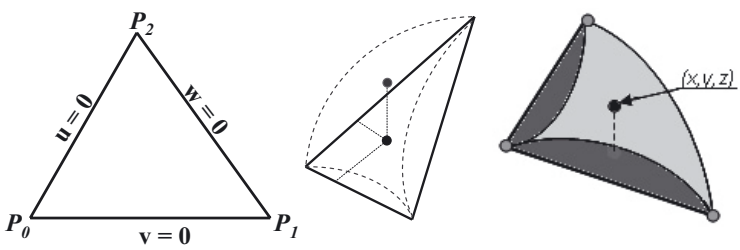

Fig. 5. Évaluation d'un point sur la surface à partir d'un triangle.
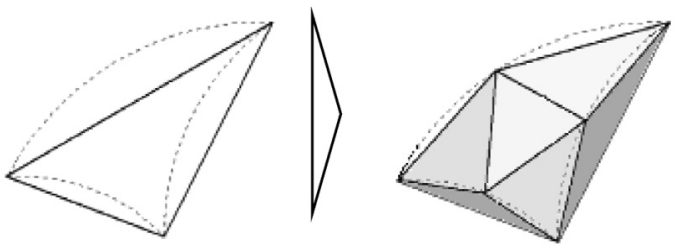

(a)

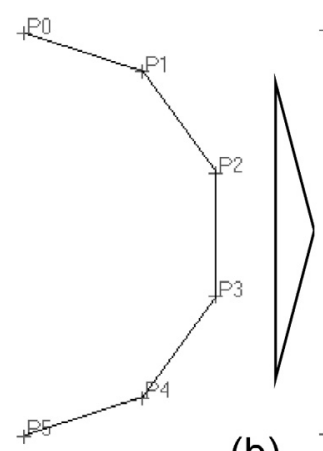

(b)

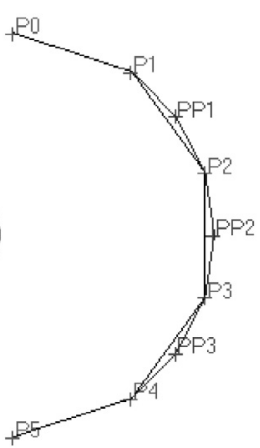

Fig. 6. (a) Subdivision successive des triangles, (b) subdivision successive des arêtes.

par déplacement des points appartenant à cette surface qui est basée sur une méthode énergétique.

Dans le même contexte d'évaluation, de paramétrisation ou de reconstruction d'une surface à partir d'une triangulation (maillage triangulaire), des travaux se sont intéressés à l'approximation d'une surface par subdivision successive du maillage correspondant [23,24] (Fig. 6). Un nouveau nœud est inséré entre deux nœuds consécutifs, afin que chaque arête soit divisée en deux et chaque triangle soit divisé en quatre triangles plus fins tout en respectant la forme de l'objet maillé. D'autres travaux sont basés sur des différents schémas de subdivisions connues (schéma de Catmull-Clark, schéma de Loop, schéma de Butterfly...), pour raffiner un maillage successivement en vue d'obtenir une surface lisse $[25,26]$.

D'autres travaux se sont intéressés à la recherche de la topologie (les orientations, les connectivités... ) des différentes entités topologiques d'un modèle BREP [27-30].

Les différents travaux développés au cours du temps, autour de l'évaluation et de la construction des courbes 


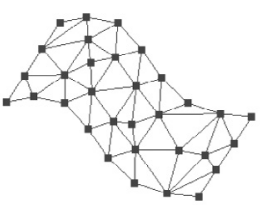

Triangulation

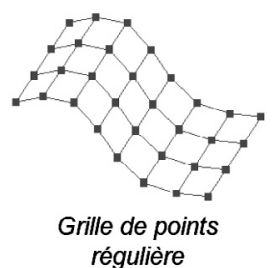

régulière

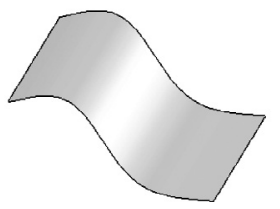

Surface reconstruite
Fig. 7. Reconstruction d'une surface à partir d'un nuage de points.

et des surfaces à partir d'un maillage triangulaire, se sont intéressés à l'évaluation de la surface localement au voisinage d'un nœud ou un triangle du maillage. Notre problématique de recherche est l'évaluation et la reconstruction de la surface porteuse d'une face du modèle CAO à partir de la triangulation correspondante. Nous avons adapté ces méthodes pour obtenir une formulation surfacique globale sous forme de NURBS.

\section{Reconstruction des entités topologiques d'un modèle BREP}

Un modèle BREP est une description géométrique et topologique des frontières d'un objet mécanique. Il est composé par des faces qui possèdent des surfaces porteuses et qui sont limitées par des contours, chacun de ces derniers est composé par des arêtes. Chaque arête possède une courbe porteuse et est limitée par deux sommets. Pour obtenir le modèle BREP, chacune de ces entités (face, arête et sommet) doit être reconstruite tout en suivant la hiérarchie mentionnée.

\subsection{Reconstruction des surfaces porteuses des faces}

La surface porteuse, d'une face reconstruite à partir d'une triangulation issue d'un maillage déformé, est dans la plupart des cas de type complexe (NURBS). La reconstruction des surfaces complexes est plus compliquée que celle des courbes. En effet, d'après la littérature et les théories des surfaces complexes (NURBS), la reconstruction nécessite une grille de points, d'interpolation ou de contrôle, organisée dans l'espace (Fig. 7) [31]. Or dans notre cas, le maillage est de type tétraédrique, donc le maillage des faces est triangulaire. La surface reconstruite doit interpoler les points de la triangulation (un nuage de points interconnectés par des segments).

L'approche de reconstruction est basée sur des outils et des fonctions disponibles dans notre environnement. L'approche est développée sous l'environnement Open Cascade (Matra Datavision) qui contient des fonctions dans ses API (application programming interface) permettant d'adhérer une surface à un ensemble de courbes et points (Fig. 8). Ces fonctions d'Open Cascade sont basées sur des méthodes énergétiques [21, 22,32]. Cela correspond bien à notre cas : la surface, qui passe par les nouds du maillage et qui adhère aux courbes de contours, est reconstruite.

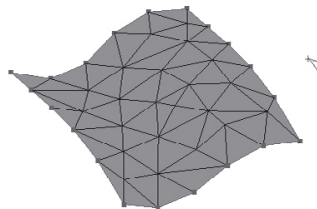

(a)

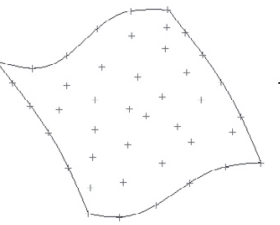

(b)

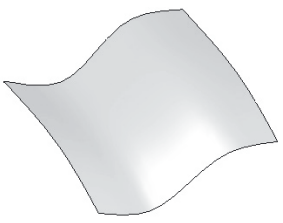

(c)
Fig. 8. (a) Maillage d'une face, (b) données extraites à partir de maillage, (c) la surface adhère sur les courbes et passe par les points.

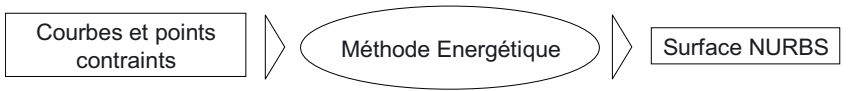

Fig. 9. Entrées/sorties de la méthode énergétique.
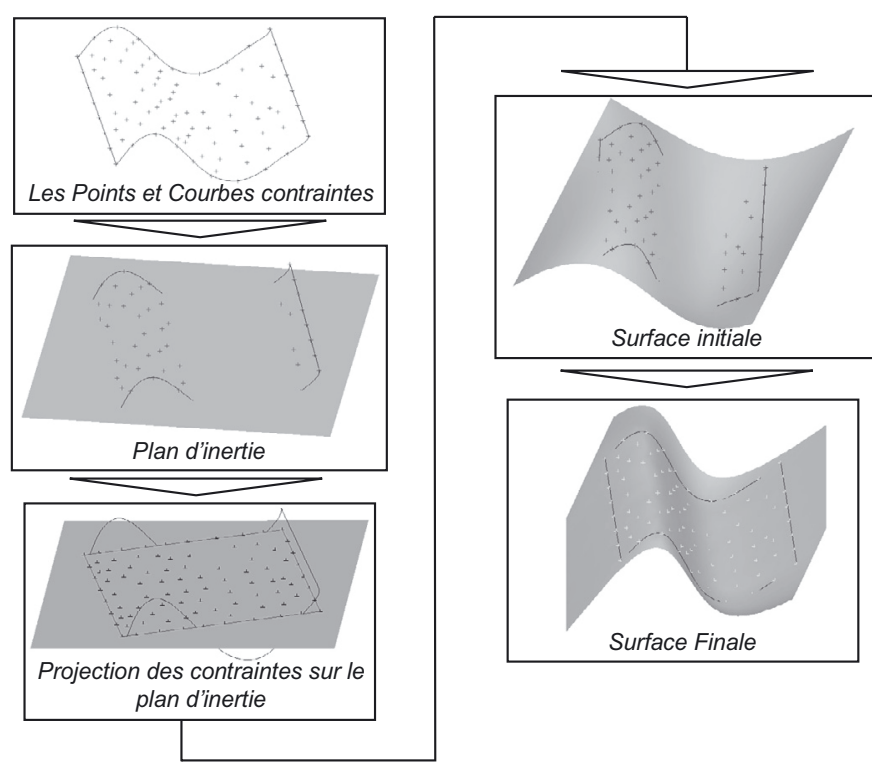

Fig. 10. Étapes de construction d'une surface NURBS à partir de la méthode énergétique.

\subsubsection{Méthode énergétique}

La méthode énergétique est une méthode développée au sein de la plateforme Open Cascade (Matra Datavision). Cette méthode possède des données (Fig. 9) :

- des courbes contraintes (ce sont des courbes reconstruites par interpolation des nœuds de maillage des arêtes dans le modèle déformé) (Fig. 8b) ;

- des points contraints (issus du maillage de la face) (Fig. 8b).

Le résultat de cette méthode est une surface (Fig. 8c) qui lisse les différentes contraintes définies précédemment.

Étant donné un ensemble de points et courbes contraints, la construction de la surface NURBS par la méthode énergétique se fait en quatre grandes étapes :

- première étape : chaque courbe contrainte est discrétisée en un ensemble de points. Le plan d'inertie de l'ensemble des points est construit. Un plan d'inertie est le plan qui passe par le flux maxi de points (Fig. 10); 


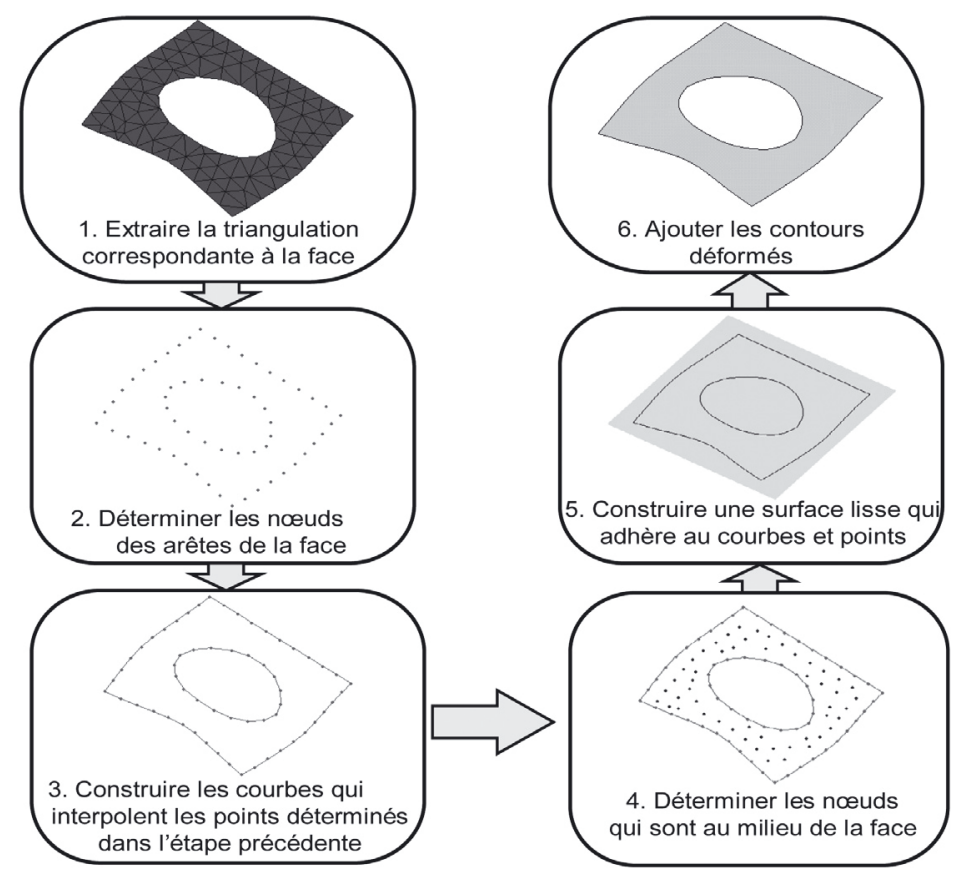

Fig. 11. Algorithme de reconstruction d'une face à partir d'un maillage par la méthode énergétique.

- deuxième étape : les différentes contraintes sont projetées sur ce plan (Fig. 10);

- troisième étape : une surface initiale qui est proche de la surface finale est calculée par déformation du plan d'inertie (Fig. 10). Cette dernière opération se fait en se basant sur un ensemble bien déterminé de points contraints, par minimisation des distances entre ces derniers et leurs projetés sur le plan d'inertie. Notons qu'à ce niveau la surface initiale peut être choisie par l'utilisateur, dans ce cas la surface donnée par ce dernier est utilisée dans l'étape suivante. On peut dire que cette étape (la troisième étape) représente une phase de préparation d'une surface pour la quatrième étape;

- quatrième étape : la surface finale est cherchée (Fig. 10) à partir de celle trouvée dans la deuxième étape. La construction de la surface se fait sur plusieurs itérations mais dans la plupart des cas une seule itération est suffisante sauf pour des surfaces plus complexes qui présentent des pics et des fortes courbures (cas peu fréquent en mécanique du solide).

\subsection{Algorithme développé}

L'algorithme de reconstruction d'une face déformée à partir d'un maillage est le suivant (Fig. 11).

\subsubsection{Synthèse}

L'approche est efficace pour la reconstruction, elle s'applique quelles que soient la nature et l'importance des déformations. Des problèmes apparaissent parfois, lors de la reconstruction, si le maillage est grossier et les informations (points contraints) ne sont pas suffisantes. Ces problèmes se manifestent par l'apparition de régions bombées et par l'apparition de pics sur la surface. Dans ce cas, pour ajouter de l'information dans quelques régions de la surface, il faut mieux raffiner le maillage ou insérer des nouveaux points calculés à partir du maillage. Ceci correspond à l'utilisation des surfaces de subdivision. Cette deuxième approche est retenue pour la reconstruction du modèle BREP.

\subsection{Reconstruction des arêtes et contours}

La reconstruction des arêtes est simple. Les noeuds qui correspondent à une arête dans le maillage déformé sont déterminés puis sont interpolés pour obtenir l'arête correspondante. Dans le cas de petites perturbations, l'arête garde sa nature de celle avant déformation (par exemple une arête circulaire reste circulaire) seuls les paramètres changent : vecteur directeur, point d'insertion... [8].

Dans le cas des déformations importantes, les points d'interpolation des arêtes, issus du maillage, sont déterminés et projetés un par un sur la surface en question. Par la suite les arêtes et contours, qui doivent être dans l'espace paramétrique de la surface, sont reconstruites une par une. À la fin, la surface doit être coupée (limitée) suivant ces contours pour obtenir la face (Fig. 11).

\section{Résultats de reconstruction}

\subsection{Reconstruction et validation en grandes déformations}

Pour valider l'algorithme de reconstruction développé, plusieurs exemples de pièces mécaniques sont traités. Ces 


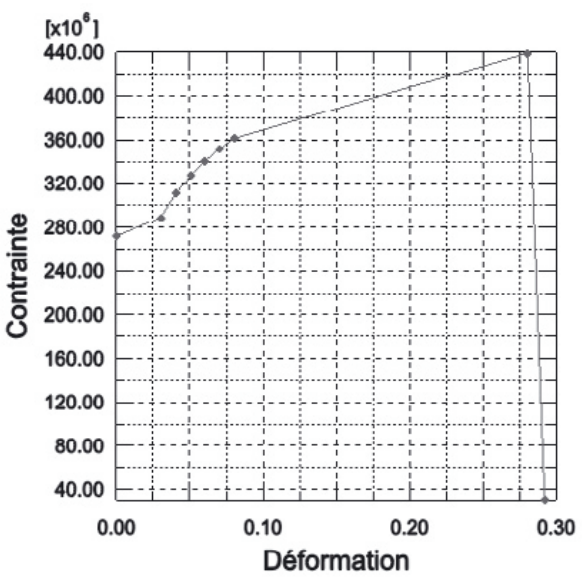

(a)

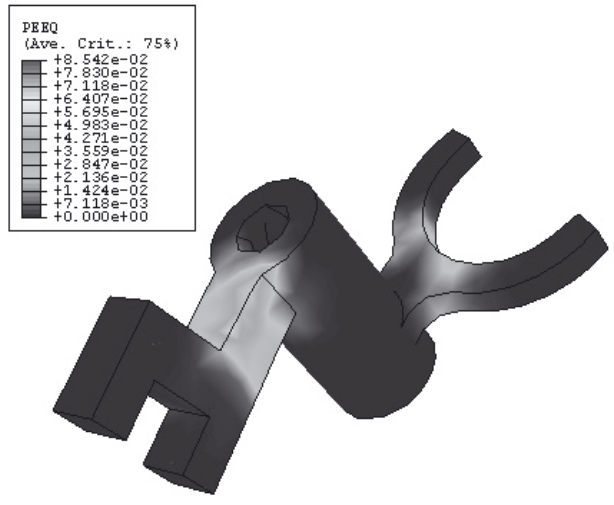

(b)

Fig. 12. (a) Courbe de contrainte en fonction de la déformation plastique de matériau utilisé, (b) début de la déformation plastique.
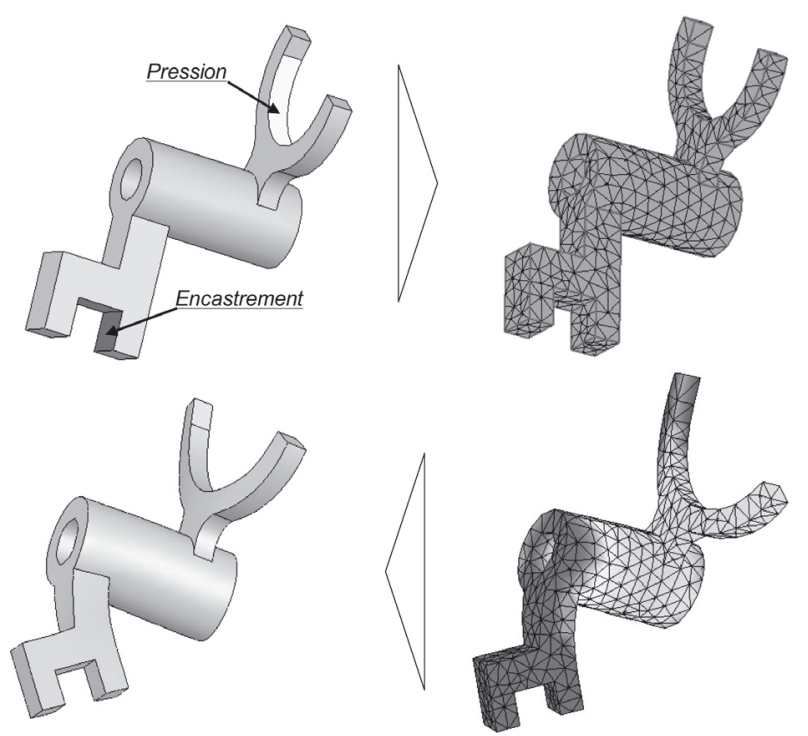

Fig. 13. Reconstruction d'une fourchette (cas d'un maillage tétraédrique linéaire).

derniers sont choisis pour leurs représentativités d'une grande classe de pièces mécaniques. Par ces exemples de validation nous avons montré que la reconstruction est faisable dans plusieurs cas (petites et grandes déformations). Ces pièces contiennent différents types de faces ainsi que différents types d'arêtes. Les faces et les arêtes sont détectées et reconstruites une par une (Sects. 5.1 et 5.2). Toutes les surfaces porteuses des faces reconstruites et les courbes porteuses des arêtes sont de types NURBS. L'erreur de reconstruction est calculée par projection des points d'interpolation (nœuds du maillage déformé) sur la surface reconstruite.

Dans l'exemple qui suit (Fig. 12), une fourchette d'embrayage est étudiée dans son domaine plastique. Le métal choisi est l'acier allié inoxydable. La courbe

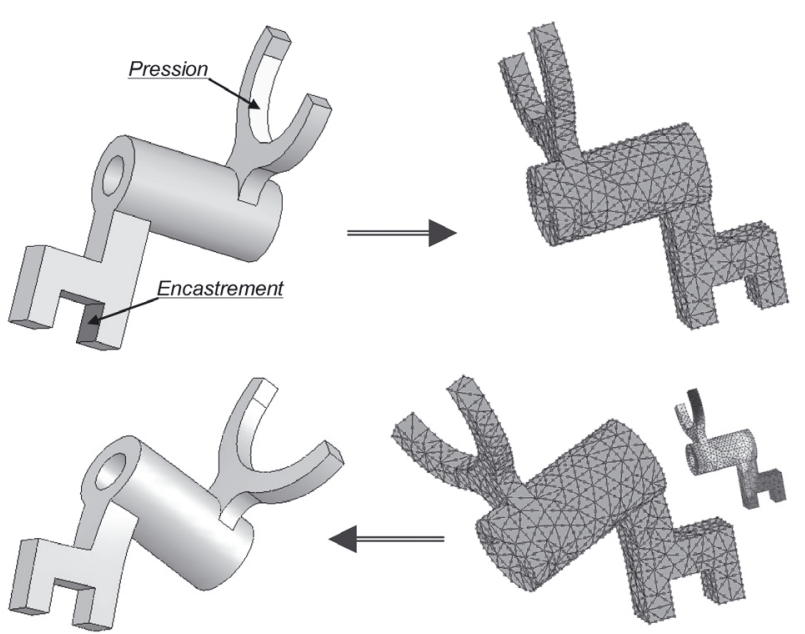

Fig. 14. Reconstruction de fourchette (maillage quadratique).

contrainte/déformation est présentée dans la figure (Fig. 12a).

Les résultats éléments-finis sont extraits après le calcul. La figure $12 \mathrm{~b}$ présente le début de la déformation plastique obtenue à une contrainte égale à 259 Mpa. La déformation plastique est obtenue à une pression de 1,7104 N.mm ${ }^{-2}$.

Pour cet exemple, deux types de maillage sont étudiés : maillage linéaire grossier (Fig. 13) et maillage quadratique (Fig. 14). Cela permet de mesurer la précision du modèle reconstruit et le temps mis pour la reconstruction, en fonction de la quantité d'information disponible pour sa reconstruction. Deux principaux facteurs indicateurs des performances de l'algorithme de reconstruction sont les suivants :

- le temps CPU de reconstruction : en augmentant le nombre de nœuds de modèle (essentiellement sur les faces et les arêtes) le temps de reconstruction augmente. Dans l'exemple de la fourchette d'embrayage, 


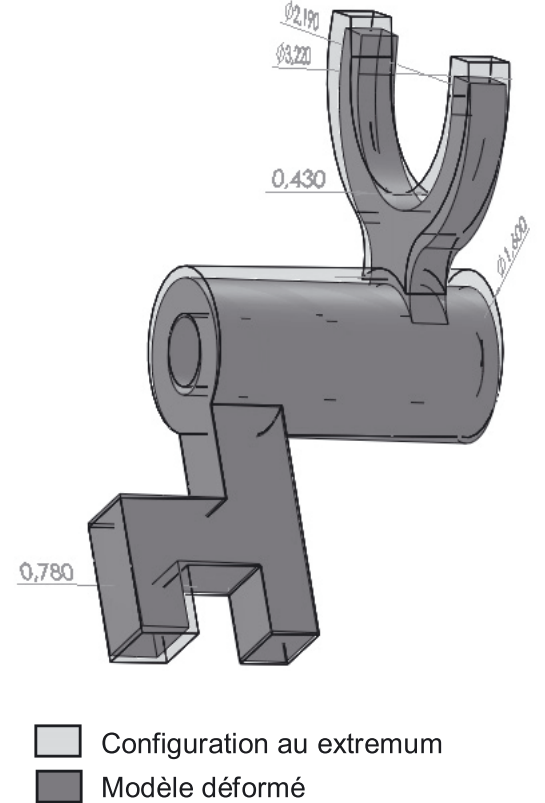

(a)

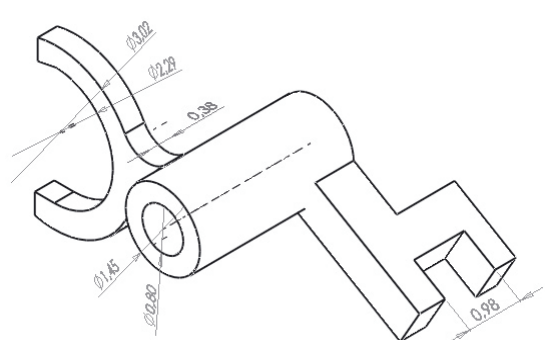

(b)

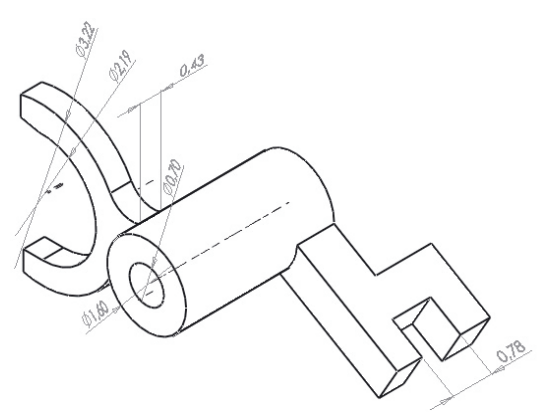

(c)

Fig. 15. (a) Validation de modèle déformé par rapport à la configuration au maximum de matière, (b) configuration au minimum de matière, (c) configuration au maximum de matière.

Tableau 1. Résultats de validation (cas de maillage linéaire).

\begin{tabular}{ll} 
Nombre de faces & 27 faces \\
Nombre de tétraèdres & 2518 tétraèdres \\
$\begin{array}{l}\text { Nombre de nœuds sur les frontières } \\
\text { (faces et arêtes) du modèle }\end{array}$ & 624 nœuds \\
Temps CPU de reconstruction & $5 \mathrm{~s}$ \\
Ordre de déplacements & $0-2,6162 \mathrm{~mm}$ \\
Erreur maximale & $81,28 \times 10^{-3} \mathrm{~mm}$ \\
\hline
\end{tabular}

Tableau 2. Résultats de validation (cas de maillage quadratique).

\begin{tabular}{ll}
\hline Nombre de faces & 27 faces \\
Nombre de tétraèdres & 1506 tétraèdres \\
$\begin{array}{l}\text { Nombre de nœuds sur les frontières } \\
\text { (faces et arêtes) du modèle }\end{array}$ & 2496 nœuds \\
Temps CPU de reconstruction & $8 \mathrm{~s}$ \\
Ordre de déplacements & $0-2,6162 \mathrm{~mm}$ \\
Erreur maximale & $50,81 \times 10^{-3} \mathrm{~mm}$ \\
\hline
\end{tabular}

le temps de reconstruction passe de $5 \mathrm{~s}$ à $8 \mathrm{~s}$ lorsque le nombre de nœuds, sur les faces et les arêtes, augmente de 624 à 2496 (Tab. 1) et (Tab. 2);

- l'erreur de reconstruction : est la distance calculée entre un point contraint et son projeté sur la surface reconstruite. Les exemples étudiés montrent que les valeurs des erreurs de reconstruction sont acceptables. Les erreurs calculées sont négligeables par rapport aux déplacements de nœuds ainsi par rapport aux dimensions de la pièce. L'exemple de la fourchette d'embrayage montre que la précision du modèle reconstruit augmente en augmentant le nombre de nœuds. Dans un premier temps, l'erreur maximale de reconstruction est de $81,28 \times 10^{-3} \mathrm{~mm}$ avec un nombre de nouds égal à 624 (Tab. 1). Dans un deuxième temps, cette erreur passe à $50,81 \times 10^{-3} \mathrm{~mm}$ lorsque le nombre de nœuds passe à 2496 (Tab. 2) en utilisant un maillage quadratique.

En modifiant le nombre de nœuds, la précision et le temps de reconstruction changent. En augmentant le nombre de nœuds le temps de reconstruction augmente et la précision augmente. En diminuant le nombre de nœuds, on gagne en temps de reconstruction et on perd en précision.

\subsection{Perspectives des travaux de validation : cas de petites déformations}

En petites déformations, lorsque la pièce est utilisée dans un assemblage mécanique, le modèle (en dimensions nominales) déformé reconstruit doit être compris entre les configurations aux extremum (au maximum et au minimum de matière) permis par les tolérances de la pièce (Fig. 15). Les travaux se poursuivent à présent pour développer un outil de prise en compte du tolérancement (dimensionnelles et géométriques) dans le modèle CAO [33-35] afin de vérifier la validité de modèle dans son état déformé. Le principe de l'indépendance $[36,37]$ exige que chaque tolérance soit traitée à part. Les figures $15 \mathrm{~b}$ et $15 \mathrm{c}$ montrent les deux configurations (au maximum et au minimum de matière) 

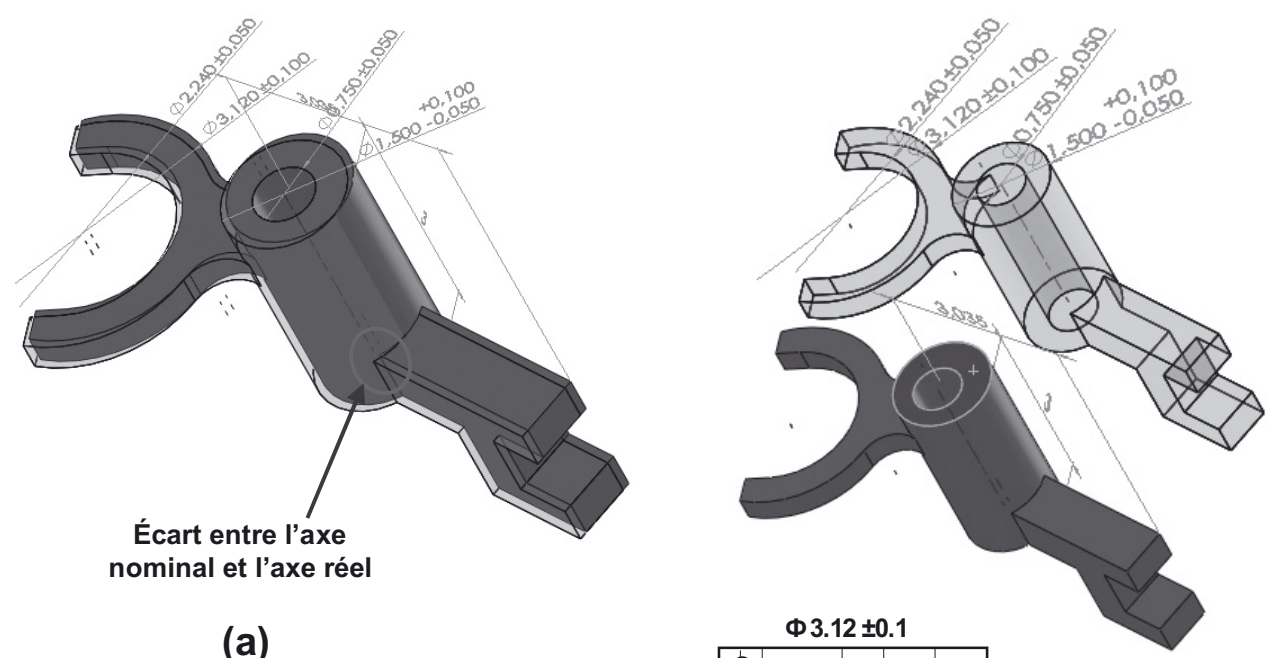

(a)

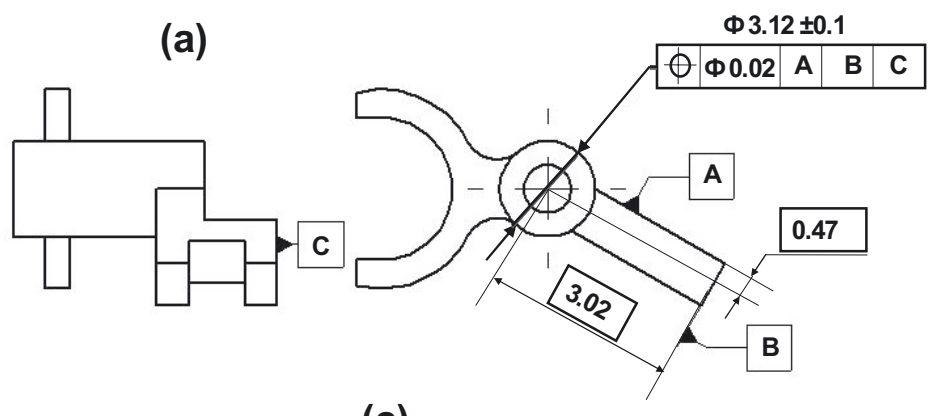

(b)

Fig. 16. (a) Validation de modèle déformé par rapport à une configuration réaliste (déduite à partir de tolérances de localisation), (b) une des configurations réalistes de la pièce, (c) tolérance géométrique.

déduites des tolérances dimensionnelles. La validation de la pièce déformée doit vérifier que chacune de ses faces doit être comprise entre celles de modèles aux extrémums (en maximum et minimum). Le contrôle des tolérances géométriques de la pièce est présenté dans la figure 16 .

\section{Conclusions et perspectives}

Dans cet article, une technique de reconstruction des entités géométriques d'un modèle BREP est présentée. L'approche présentée traite la partie géométrique du modèle BREP et suppose que la topologie du modèle n'a pas changé après déformation (le nombre des faces, arêtes et sommets et leurs connectivités restent les mêmes). Les travaux se poursuivent à présent en solutionnant certaines limitations :

- les erreurs numériques lors de la reconstruction qui empêchent la fermeture de modèle BREP reconstruit;

- l'outil de reconstruction peut être amélioré pour être plus dynamique. Cela rend la simulation numérique faisable en temps réel.

\section{Références}

[1] N. Aifaoui, Intégration CAO/Calcul, une approche par les features de calcul, Thèse de Doctorat, Université de Valenciennes, juillet 2003
[2] S. Tichkiewitch, De la CFAO à la conception intégrée, Int. J. CADCAM and Comp. Graph. 9 (1994) 609-621

[3] N. Troussier, Contribution à l'intégration du calcul mécanique dans la conception des produits techniques, proposition méthodologique pour l'utilisation et la réutilisation, Thèse de Doctorat, Université Joseph Fourier, Grenoble 1, 1999

[4] S. Menand, Modélisation pour la réutilisation du processus Multi-acteurs de produits industriels, application à la conception fonctionnelle des systèmes de direction, Thèse de Doctorat de l'Institut National Polytechnique de Grenoble, janvier 2002

[5] A. Benamara, Contribution à l'intégration de la composante calcul dans une démarche de conception fonctionnelle intégrée, Thèse de doctorat, Université de Valenciennes, décembre 1998

[6] J. Sam, Constraint consistency techniques for continuous domains, Thèse de doctorat, École polytechnique fédérale de Lausanne, 1995

[7] V. François, Méthodes de maillage et de remaillage automatiques appliquées à la modification de modèle dans le contexte de l'ingénierie simultanée, Thèse de doctorat, Université Henri Poincaré, Nancy 1, novembre 1998

[8] B. Louhichi, A. BenAmara, V. François, Intégration $\mathrm{CAO} /$ Calcul par reconstruction des modèles $\mathrm{CAO}$ à partir des résultats de calcul, Revue Int. d'Ingénierie Numérique 1 (2005) 9-26

[9] L. Fine, Processus et méthodes d'adaptation et d'idéalisation de modèles dédiés à l'analyse de structures mécaniques, Thèse de doctorat, Institut national polytechnique de Grenoble, 2001 
[10] M. Tollenaere, Conception des produits en mécanique, HERMES 1998, ISBN2-86601-694-7

[11] E.M. Mortenson, Geometric Modeling, Wiley, New York, 1985

[12] J.-P. Kruth, A. Kerstens, Reverse engineering modelling of free-form surfaces from point clouds subject to boundary conditions, J. Mat. Proc. Tech. 76 (1998) 120-127

[13] Z. Yin, Reverse engineering of a NURBS surface from digitized points subject to boundary conditions, Computer \& Graphics 28 (2004) 207-212

[14] L.A. Piegl, W. Tiller, The NURBS book, Springer-Verlag, 1997

[15] H.B. Jung, K. Kim, A New Parameterisation Method for NURBS Surface Interpolation, Int. J. Adv. Manufacturing Technology 16 (2000) 784-790

[16] D.J. Walton, D.S. Meek, A triangular G1 patch from boundary curves, Computer Aided Design 28 (1996) 113123

[17] P. Kashyap, Geometric interpretation of continuity over triangular domains, Computer Aided Geometric Design 15 (1998) 773-786

[18] J.M. Morvan, B. Thibert, On the approximation of a smooth surface with a triangular mesh, Computational Geometry 23 (2002) 337-352

[19] S. Wang, A smooth surface interpolation to 3D triangulations, J. Comp. Appl. Math. 163 (2004) 287-293

[20] S.J. Owen, D.R. White, T.J. Tautges, Facet-based surfaces for 3D, mesh generation, 11th international meshing roundtable, Ithaca, New York, 2002

[21] O. Volpin, A. Sheffer, M. Bercovier, Joskowicz, Mesh simplification with smooth surface reconstruction, Computer Aided Design 30 (1998) 875-882

[22] R.F. Sarraga, Modifying CAD/CAM surfaces according to displacements prescribed at a finite set of points, Computer Aided Design 36 (2004) 343-349

[23] B.Y. Ren, I. Hagiwara, Composite freeform surface reconstruction using recursive interpolating subdivision scheme, Computers in Industry 50 (2003) 265-275

[24] X. Yang, Surface interpolation of meshes by geometric subdivision, Computer Aided Design 37 (2005) 497-508
[25] M. Weiyin, M. Xiaohu, S.-K. Tso, Z. Pan, A direct approach for subdivision surface fitting from a dense triangle mesh, Computer Aided Design 36 (2004) 525-536

[26] D. Rypl, Z. Bittnar, Triangulation of 3D surfaces reconstructed by interpolating subdivision, Computers and Structures 82 (2004) 2093-2103

[27] P. Krysl, M. Ortiz, Extraction of boundary representation from surface triangulations, Int. J. Num. Meth. Eng. 50 (2001) 1737-1758

[28] J.C. Park, Y.C. Chung, A tolerant approach to reconstruct topology from unorganized trimmed surfaces, Computer Aided Design 35 (2003) 807-812

[29] S.J. Owen, D.R. White, Mesh based geometry: A systematic approach to constructing geometry from a finite element mesh, 10th International Meshing Roundtable Newport Beach, California, USA, 2001

[30] S.J. Owen, D.R. White Mesh-based geometry, Int. J. Num. Meth. Eng. 58 (2003) 375-395

[31] J.C. Léon, Modélisation des courbes et des surfaces pour la CFAO, Hermes, Paris

[32] (Opencascade) www.Opencascade.com

[33] M. Tlija, A. Benamara, V. Francois, Modèle de prise en compte des tolérances en $\mathrm{CAO}$, actes du premier congrès Tunisien de Mécanique, 2008, Hammamet, 17-19 mars 2008

[34] M. Tlija, B. Louhichi, A. Benamara, A. Riviere, Modélisation et simulation réalistes d'un assemblage mécanique, CMSM09, Hammamet, 16-18 mars 2009

[35] C. Mehdi-Souzani, B. Anselmetti, Integration of automatic functional tolerancing into the design process, Proceedings of IDMME-Virtual Concept 2008, Beijing (China), 08-10 October 2008

[36] Tolérancement géométrique des produits, L. Mathieu, F. Villeneuve (éds.), éditions Hermes-Lavoisier, ISBN 9782-7462-1509-2,3 p. 79, avril 2007

[37] P. Bourdet, Spécification Géométrique des Produits (GPS), Université de Paris VI-ENS de Cachan, février 2001 